\title{
NONNEGATIVE LINEARIZATION FOR ORTHOGONAL POLYNOMIALS WITH EVENTUALLY CONSTANT JACOBI PARAMETERS
}

\author{
WOJCIECH MŁOTKOWSKI \\ Institute of Mathematics, Wroctaw University \\ Pl. Grunwaldzki 2/4, 50-384 Wrocław, Poland \\ E-mail:mlotkow@math.uni.wroc.pl
}

\begin{abstract}
We study the nonnegative product linearization property for polynomials with eventually constant Jacobi parameters. For some special cases a necessary and sufficient condition for this property is provided.
\end{abstract}

1. Introduction. Let $\left\{P_{n}\right\}_{n=0}^{\infty}$ be a sequence of polynomials defined by the following recurrence relation: $P_{0}(x)=1$ and for $n \geq 0$

$$
x P_{n}(x)=P_{n+1}(x)+\beta_{n} P_{n}(x)+\gamma_{n-1} P_{n-1}(x)
$$

(under the convention that $P_{-1}(x)=0$ ), where $\left\{\gamma_{n}\right\}_{n=0}^{\infty}$ and $\left\{\beta_{n}\right\}_{n=0}^{\infty}$ are sequences of real numbers, called Jacobi parameters, and $\gamma_{n}>0$. Then all $P_{n}$ are monic and $\operatorname{deg} P_{n}=n$. We denote by $\mathcal{L}$ the linear functional on $\mathbb{R}[x]$ defined by: $\mathcal{L}\left(P_{0}\right)=1$ and $\mathcal{L}\left(P_{n}\right)=0$ for $n \geq 1$. Then we have

$$
\mathcal{L}\left(P_{m} P_{n}\right)=\delta_{m, n} \cdot \gamma_{0} \gamma_{1} \ldots \gamma_{m-1} .
$$

In view of Favard's theorem $[5] \mathcal{L}$ can be expressed as an integral with respect to a probability measure on the real line.

Our aim is to study the linearization coefficients which are uniquely defined by

$$
P_{m}(x) P_{n}(x)=\sum_{j} c(j, m, n) P_{j}(x) .
$$

It is known [2] that many classical orthogonal polynomials admit nonnegative product linearization, i.e. all $c(j, m, n)$ are nonnegative even though their exact values can be unknown. In this case one can define a hypergroup structure [2] on the set of nonnegative

2000 Mathematics Subject Classification: $42 \mathrm{C} 05$.

Key words and phrases: orthogonal polynomials, linearizing coefficients.

The paper is in final form and no version of it will be published elsewhere. 
integers by putting

$$
\delta_{m} * \delta_{n}:=\sum_{k} \frac{c(k, m, n) P_{k}\left(x_{0}\right)}{P_{m}\left(x_{0}\right) P_{n}\left(x_{0}\right)} \delta_{k},
$$

where the normalizing point $x_{0}$ is choosen such that $x_{0} \geq \sup (\operatorname{supp} \mu)$, so that $P_{m}\left(x_{0}\right)>0$ for all $m$. Extending this to convex combinations one obtains an associative and commutative convolution on the class of probability measures on the set $\{0,1,2, \ldots\}$. There are also some general criteria for nonnegative product linearization, stated in terms of the parameters $\gamma_{n}, \beta_{n}$ [1, 9, 11].

Multiplying both sides of $(3)$ by $P_{k}$ and applying $\mathcal{L}$ we get

$$
\mathcal{L}\left(P_{k} P_{m} P_{n}\right)=c(k, m, n) \gamma_{0} \gamma_{1} \ldots \gamma_{k-1} .
$$

We denote $L(k, m, n):=\mathcal{L}\left(P_{k} P_{m} P_{n}\right)$ and $\Gamma(k):=\gamma_{0} \gamma_{1} \ldots \gamma_{k-1}$.

The following properties of the coefficients $L(k, m, n)$ are easy to verify [9]:

$$
\begin{aligned}
L(k, m, k+m)= & \Gamma(k+m), \\
L\left(k_{1}, k_{2}, k_{3}\right)= & L\left(k_{\sigma_{1}}, k_{\sigma_{2}}, k_{\sigma_{3}}\right) \\
L(k, m, n)= & 0 \quad \text { whenever } n>k+m, \\
L(k, m, n)= & L(k-1, m, n+1)+\left(\beta_{n}-\beta_{k-1}\right) L(k-1, m, n) \\
& +\gamma_{n-1} L(k-1, m, n-1)-\gamma_{k-2} L(k-2, m, n)
\end{aligned}
$$

for any $k, m, n$ and any permutation $\sigma$ of the set $\{1,2,3\}$.

In particular, one can check that for $1 \leq k \leq m$ we have:

$$
L(k, m, k+m-1)=\Gamma(k+m-1)\left[\sum_{i=0}^{k-1}\left(\beta_{m+i}-\beta_{i}\right)\right]
$$

and

$$
\begin{aligned}
& L(k, m, k+m-2)=\Gamma(k+m-2) \\
& \times\left[\gamma_{m-1}+\sum_{i=0}^{k-2}\left(\gamma_{m+i}-\gamma_{i}\right)+\sum_{0 \leq i<j \leq k-1}\left(\beta_{m+i}-\beta_{i}\right)\left(\beta_{m+j-1}-\beta_{j}\right)\right] .
\end{aligned}
$$

2. Orthogonal polynomials with eventually constant Jacobi parameters. From now on we will assume that the coefficients $\gamma_{n}, \beta_{n}$ are constant from some place. Orthogonal polynomials of this kind and the corresponding probability measures are sometimes encountered in various limit theorems in noncommutative probability [3, 7]. The basic example is the Wigner measure

$$
\mu=\frac{1}{2 \pi \gamma} \sqrt{4 \gamma-(t-\beta)^{2}} d t
$$

on $[\beta-2 \sqrt{\gamma}, \beta+2 \sqrt{\gamma}]$, having both sequences $\gamma_{n} \equiv \gamma, \beta_{n} \equiv \beta$ constant, which plays the role of the Gaussian measure in free probability.

Assumption. For $k \geq M$ we have $\beta_{k}=\beta, \gamma_{k}=\gamma$.

The case $M=1$ is quite interesting. For example, the related four parameter family of measures is closed under the free and boolean powers [6, 10] and $\left\{P_{n}\right\}$ admits nonnegative product linearization if and only if $\gamma_{0} \leq 2 \gamma$ and $\beta_{0} \leq \beta$ [8. This class of probability 
measures contains the Marchenko-Pastur measures (where $\gamma_{0}=\gamma, \beta_{0}=\gamma, \beta=\gamma+1$ ), which in free probability play the role of Poisson measures, as well as the limit measures related to conditional freeness (with $\gamma_{0}=a^{2}, \gamma=b^{2}, \beta_{0}=\beta=0$ in the central limit theorem, and with $\gamma_{0}=a, \gamma=b, \beta_{0}=a, \beta=b+1$ in the Poisson limit theorem [3]).

We will base on the following lemma, which will be used together with relation (7).

LEMma 2.1. If $n+k-m>2 M$ then $L(k, m, n)=\gamma L(k-1, m, n-1)$.

Proof. Without loss of generality we can assume that $k \leq n$. We proceed by induction on $j:=k+m-n$. Note that then

$$
2 k>2 M+j
$$

because

$$
n>2 M+m-k=2 M+(n+j-k)-k=2 M-2 k+j+n .
$$

In particular, $k>M$ and hence $\beta_{n}-\beta_{k-1}=\beta-\beta=0$, so we can ignore the second summand in 9 .

If $j=0$ then $n=k+m$ and

$$
L(k, m, k+m)=\Gamma(k+m)=\gamma \Gamma(k+m-1)=\gamma L(k-1, m, k+m-1) .
$$

If $j=1$ then, by 13$), M<k \leq n$. Since $n=k+m-1$ we have $L(k-1, m, n+1)=$ $L(k-2, m, n)=0$. Then, by $(9)$, we get $L(k, m, n)=\gamma L(k-1, m, n-1)$.

Finally, assume that $j \geq 2$. Then $k>M+1$ and hence $\gamma_{k-2}=\gamma$. Therefore, by induction, we have $L(k-1, m, n+1)=\gamma L(k-2, m, n)$ so in (9) the first summand cancels with the last one, so we get $L(k, m, n)=\gamma L(k-1, m, n-1)$, which concludes the proof.

Definition 2.2. We write $\left(k^{\prime}, m^{\prime}, n^{\prime}\right) \overrightarrow{\mathcal{R}}_{0}(k, m, n)$ if either

$$
\begin{aligned}
& k^{\prime}=k, m^{\prime}=m-1, n^{\prime}=n-1 \text { and } m+n-k>2 M \text { or } \\
& k^{\prime}=k-1, m^{\prime}=m, n^{\prime}=n-1 \text { and } k+n-m>2 M \text { or } \\
& k^{\prime}=k-1, m^{\prime}=m-1, n^{\prime}=n \text { and } k+m-n>2 M .
\end{aligned}
$$

Denote by $\overrightarrow{\mathcal{R}}$ be the smallest reflexive and transitive relation containing $\overrightarrow{\mathcal{R}}_{0}$. Since $\left(k^{\prime}, m^{\prime}, n^{\prime}\right) \overrightarrow{\mathcal{R}}_{0}(k, m, n)$ implies $k^{\prime} \leq k, m^{\prime} \leq m, n^{\prime} \leq n$ we see that $\overrightarrow{\mathcal{R}}$ is weakly antisymmetric and hence is a partial order. Note also that if $\left(k^{\prime}, m^{\prime}, n^{\prime}\right) \overrightarrow{\mathcal{R}}(k, m, n)$ then $k+m+n-k^{\prime}-m^{\prime}-n^{\prime}:=2 r$ is even and, in view of the Lemma 2.1,

$$
L(k, m, n)=\gamma^{r} L\left(k^{\prime}, m^{\prime}, n^{\prime}\right) .
$$

Therefore it is sufficient to examine $L(k, m, n)$ for those triples $(k, m, n)$ which satisfy $0<k \leq m \leq n<k+m$ and $m+n-k \leq 2 M$. The set of such triples will be denoted by $\Sigma$.

Proposition 2.3. The number of elements in $\Sigma$ is equal to

$$
\frac{M(M+1)(M+2)}{3} \text {. }
$$

Proof. Set $r:=k+m-n, 1 \leq r \leq 2 M$. Then the inequalities $k \leq m \leq n$ and $m+n-k \leq 2 M$ are equivalent to

$$
r \leq k \leq m \leq M+\frac{r}{2} .
$$


Note that for fixed integers $r \leq s$ there are exactly

$$
\frac{(s-r+1)(s-r+2)}{2}
$$

pairs $(k, m)$ such that $r \leq k \leq m \leq s$. Therefore for fixed $r$ there are exactly

$$
\frac{(M-t+1)(M-t+2)}{2}
$$

pairs $(k, m)$ satisfying $r \leq k \leq m \leq M+r / 2$, where either $r=2 t$ or $r=2 t-1$. Now we have

$$
\sum_{t=1}^{M}(M-t+1)(M-t+2)=\sum_{s=1}^{M} s(s+1)=\frac{M(M+1)(M+2)}{3}
$$

which completes the proof.

Let $\mathcal{R}$ be the smallest equivalence relation containing $\overrightarrow{\mathcal{R}}$. Now we are going to describe the elements $(k, m, n)$ of $\Sigma$ and their $\mathcal{R}$-equivalence classes. We assume that $0<k \leq m \leq$ $n<k+m, m+n-k \leq 2 M$ and put $r:=k+m-n$. We have $1 \leq r \leq 2 M$. If $r$ is even we put $r=2 t$, otherwise $r=2 t-1$, so that $1 \leq t \leq M$.

Case 0. If $m+n+2-k \leq 2 M$ then $(k, m, n)$ has no successor, so $[(k, m, n)]_{\mathcal{R}}=$ $\{(k, m, n)\}$. Applying Proposition 2.3 to $M-1$ we see that the number of such triples is $(M-1) M(M+1) / 3$.

Case 1. Assume that $m+n+2-k>2 M$ and $k+n+2-m \leq 2 M$. The former, together with the third inequality in (15) leads to $m=M+\left[\frac{r}{2}\right]$. Hence, we have to choose $r$ and $k$ such that $1 \leq r \leq 2 M$ and $r \leq k<M+\left[\frac{r}{2}\right]$, which gives $M(M-1)$ choices. Note that in this case, for $s \geq 0$ the only successor of $(k, m+s, n+s)$ is $(k, m+s+1, n+s+1)$. Therefore

$$
[(k, m, n)]_{\mathcal{R}}=\{(k, m+s, n+s): s \geq 0\} .
$$

Case 2. Assume that $k+n+2-m>2 M$ and $k+m+2-n \leq 2 M$. Then the former, together with 15 , leads to $k=m=M+\left[\frac{r}{2}\right]$ (hence $n=2 M-1$ if $r$ is odd and $n=2 M$ if $r$ is even), while the latter means that $r \leq 2 M-2$. Hence $k=m=M+t-\epsilon$, $n=2 M-\epsilon, \epsilon \in\{0,1\}, 1 \leq t \leq M-1$. This leads to $2 M-2$ classes:

$$
\begin{gathered}
{[(M+t-\epsilon, M+t-\epsilon, 2 M-\epsilon)]_{\mathcal{R}}} \\
=\{(M+t+p-\epsilon, M+t+q-\epsilon, 2 M+p+q-\epsilon): p, q \geq 0\} \\
=\{(a, b, a+b-2 t+\epsilon): a, b \geq M+t-\epsilon\} .
\end{gathered}
$$

Case 3. Finally, assume that $r=k+m-n>2 M-2$. Then (15) implies that $r=2 M-\epsilon$, $\epsilon \in\{0,1\}$, and $r=k=m=n$, which leads to two classes:

$$
\begin{gathered}
{[(2 M-\epsilon, 2 M-\epsilon, 2 M-\epsilon)]_{\mathcal{R}}} \\
=\{(2 M-\epsilon+q+s, 2 M-\epsilon+p+s, 2 M-\epsilon+p+q): p, q, s \geq 0\} \\
=\{(a, b, c): a+b-c, a+c-b, b+c-a \geq 2 M-\epsilon, \text { and } a+b+c-\epsilon \text { is even }\} .
\end{gathered}
$$

We will denote by $\Sigma_{i}$ the set of those triples in $\Sigma$ which fall to Case $i$. 
2.1. The case $M=2$. Let us apply our results to the case when $M=2$. Interesting examples of polynomials of this kind and the corresponding measures were studied in [7]. We have

$$
\begin{array}{ll}
\Sigma_{0}=\{(1,1,1),(2,2,2)\}, & \Sigma_{1}=\{(1,2,2),(2,3,3)\}, \\
\Sigma_{2}=\{(2,2,3),(3,3,4)\}, & \Sigma_{3}=\{(3,3,3),(4,4,4)\} .
\end{array}
$$

Assume that

$$
\gamma_{n}=\left\{\begin{array}{ll}
a & \text { if } n=0, \\
b & \text { if } n=1, \\
c & \text { if } n \geq 2,
\end{array} \quad \beta_{n}= \begin{cases}u & \text { if } n=0 \\
v & \text { if } n=1 \\
w & \text { if } n \geq 2\end{cases}\right.
$$

THEOREM 2.4. If (16) holds then we have:

o1) $L(1,1,1)=a(v-u)$,

o2) $L(1, m, m)=\Gamma(m)(w-u)$ if $m \geq 2$,

o3) $L(k, m, k+m-1)=\Gamma(k+m-1)(2 w-u-v)$ if $2 \leq k \leq m$,

o4) $L(k, m, n)=\Gamma(s-1)(2 c(2 w-u-v)-b(w-u))$ if $3 \leq k \leq m \leq n \leq k+m-3$ and $k+m+n=2 s+1$,

e1) $L(2,2,2)=a b(c+b-a+(w-u)(w-v))$,

e2) $L(2, m, m)=\Gamma(m)(2 c-a+(w-u)(w-v))$ if $m \geq 3$,

e3) $L(k, m, k+m-2)=\Gamma(k+m-2)(3 c-a-b+(w-u)(w-v))$ if $3 \leq k \leq m$,

e4) $L(k, m, n)=\Gamma(s-1)(4 c-a-2 b+(w-u)(w-v))$ if $4 \leq k \leq m \leq n \leq k+m-4$ and $k+m+n=2 s$.

Proof. The formulas (o1), (o2), (o3) and (e1), (e2), (e3) are consequences of (10) and (11) respectively. Having them, we can use (9) to compute $L(3,3,3)$ and $L(4,4,4)$. Now we apply Lemma 2.1 to conclude the proof.

COROLlary 2.5. Assuming (16), the polynomials $\left\{P_{n}\right\}$ admit nonnegative product linearization if and only if the following inequalities hold:

$$
\begin{aligned}
u & \leq v, \\
u & \leq w, \\
u+v & \leq 2 w, \\
b(w-u) & \leq 2 c(2 w-u-v), \\
0 & \leq c+b-a+(w-u)(w-v), \\
0 & \leq 4 c-a-2 b+(w-u)(w-v) .
\end{aligned}
$$

In particular, if in addition $v=w$ then the nonnegative product linearization holds if and only if $u \leq v, b(v-u) \leq 2 c(v-u), a \leq b+c$ and $2 b+a \leq 4 c$.

Proof. To avoid the inequalities related to (e2) and (e3) we use the fact that if the first and the last element of a finite arithmetic sequence are nonnegative then all its elements are nonnegative. 
2.2. The case $M=3$. If $M=3$ then we have

$$
\begin{gathered}
\Sigma_{0}=\{(1,1,1),(1,2,2),(2,2,3),(3,3,3),(2,2,2),(2,3,3),(3,3,4),(4,4,4)\}, \\
\Sigma_{1}=\{(1,3,3),(2,3,4),(3,4,4),(2,4,4),(3,4,5),(4,5,5)\}, \\
\Sigma_{2}=\{(3,3,5),(4,4,5),(4,4,6),(5,5,6)\} \\
\Sigma_{3}=\{(5,5,5),(6,6,6)\} .
\end{gathered}
$$

Now we assume that:

$$
\gamma_{n}=\left\{\begin{array}{l}
a \text { if } n=0, \\
b \text { if } n=1, \\
c \text { if } n=2, \\
d \text { if } n \geq 3,
\end{array} \quad \beta_{n}= \begin{cases}u & \text { if } n=0, \\
v & \text { if } n=1, \\
w & \text { if } n=2, \\
z & \text { if } n \geq 3 .\end{cases}\right.
$$

THEOREM 2.6. Assuming (17) we have

o1) $L(1,1,1)=a(v-u)$,

o2) $L(1,2,2)=a b(w-u)$

o3) $L(1, m, m)=\Gamma(m)(z-u)$ if $m \geq 3$,

o4) $L(2,2,3)=a b c(w+z-u-v)$,

o5) $L(2, m, m+1)=\Gamma(m+1)(2 z-u-v)$ if $m \geq 3$,

o6) $L(k, m, k+m-1)=\Gamma(k+m-1)(3 z-u-v-w)$ if $3 \leq k \leq m$,

o7) $L(3,3,3)=a b c(a(w-z)+b(u-z)+c(2 z-u-v)+d(3 z-u-v-w)$ $+(z-u)(z-v)(z-w))$,

o8) $L(3, m, m)=\Gamma(m)(a(w-z)+b(u-z)+2 d(3 z-u-v-w)+(z-u)(z-v)(z-w))$ if $m \geq 4$,

o9) $L(k, m, k+m-3)=\Gamma(k+m-3)(a(w-z)+b(u-z)+c(u+v-2 z)+3 d(3 z-$ $u-v-w)+(z-u)(z-v)(z-w))$ if $4 \leq k \leq m$,

o10) $L(k, m, n)=\Gamma(s-2)(a(w-z)+b(u-z)+2 c(u+v-2 z)+4 d(3 z-u-v-w)+$ $(z-u)(z-v)(z-w))$ if $m \geq 4$, if $5 \leq k \leq m \leq n \leq k+m-5, k+m+n=2 s-1$,

e1) $L(2,2,2)=a b(c+b-a+(w-u)(w-v))$,

e2) $L(2,3,3)=a b c(c+d-a+(z-u)(z-v))$,

e3) $L(2, m, m)=\Gamma(m)(2 d-a+(z-u)(z-v))$ if $m \geq 4$,

e4) $L(3,3,4)=a b c d(c+2 d-a-b+(z-u)(z-v)+(z-u)(z-w)+(z-v)(z-w))$,

e5) $L(3, m, m+1)=\Gamma(m+1)(3 d-a-b+(z-u)(z-v)+(z-u)(z-w)+(z-v)(z-w))$ if $m \geq 4$,

e6) $L(k, m, k+m-2)=\Gamma(k+m-2)(4 d-a-b-c+(z-u)(z-v)$ $+(z-u)(z-w)+(z-v)(z-w))$ if $4 \leq k \leq m$,

e7) $L(4,4,4)=a b c d(a c+d(5 d-2 a-2 b-c)-c(z-u)(z-v)+2 d((z-u)(z-v)+$ $(z-u)(z-w)+(z-v)(z-w)))$

e8) $L(4, m, m)=\Gamma(m)(a c+d(6 d-2 a-2 b-2 c)-c(z-u)(z-v)+2 d((z-u)(z-v)+$ $(z-u)(z-w)+(z-v)(z-w))$ if $m \geq 5$,

e9) $L(k, m, k+m-4)=\Gamma(k+m-4)(a c+d(7 d-2 a-2 b-3 c)-c(z-u)(z-v)+$ $2 d((z-u)(z-v)+(z-u)(z-w)+(z-v)(z-w))$ if $5 \leq k \leq m$,

e10) $L(k, m, n)=\Gamma(s-2)(a c+d(8 d-2 a-2 b-4 c)-c(z-u)(z-v)+2 d((z-u)(z-v)$ $+(z-u)(z-w)+(z-v)(z-w))$ if $6 \leq k \leq m \leq n \leq k+m-6, k+m+n=2 s$. 
Proof. The formulas (o1)-(o6) and (e1)-(e6) are consequences of (10) and (11). Then, using $(9)$, we compute $L(3,3,3), L(3,4,4)$ and $L(4,4,5)$, which leads to (o7), (o8) and (o9). Using these we get $L(5,5,5)$ and hence (o10). In the same way we prove (e1)-(e10).

In the same way as before we obtain

COROLlaRY 2.7. If we assume (17) then the polynomials $\left\{P_{n}\right\}$ admit nonnegative product linearization if and only if the following inequalities hold:

$$
\begin{aligned}
u & \leq v, \\
u & \leq w, \\
u & \leq z \\
u+v & \leq w+z, \\
u+v & \leq 2 z \\
u+v+w & \leq 3 z \\
a+w)+b(z-u) & \leq d(3 z-u-v-w)+c(2 z-u-v)+(z-u)(z-v)(z-w), \\
a(z-w) & \leq c+d+(z-u)(z-v), \\
a(z-w)+b(z-u) & \leq 4 d(3 z-u-v-w)-2 c(2 z-u-v)+(z-u)(z-v)(z-w), \\
a+b & \leq 2 d+c+(z-u)(z-v)+(z-u)(z-w)+(z-v)(z-w), \\
a+b & \leq 4 d-c+(z-u)(z-v)+(z-u)(z-w)+(z-v)(z-w), \\
d(2 a+2 b+c-5 d) & \leq a c+(2 d-c)(z-u)(z-v)+2 d(2 z-u-v)(z-w), \\
d(2 a+2 b+4 c-8 d) & \leq a c+(2 d-c)(z-u)(z-v)+2 d(2 z-u-v)(z-w) . \text { - }
\end{aligned}
$$

Acknowledgments. This research supported by KBN grant 1P03A 01330, by a Marie Curie Transfer of Knowledge Fellowship of the European Community's Sixth Framework Programme MTKD-CT-2004-013389, by 7010 POLONIUM project "Non-Commutative Harmonic Analysis with Applications to Operator Spaces, Operator Algebras and Probability" and by joint PAN-JSPS project: "Noncommutative harmonic analysis on discrete structures with applications to quantum probability".

\section{References}

[1] R. Askey, Linearization of the product of orthogonal polynomials, in: Problems in Analysis, R. Gunning (ed.), Princeton University Press, Princeton, NJ, 1970, 223-228.

[2] W. R. Bloom and H. Heyer, Harmonic Analysis of Probability Measures on Hypergroups, de Gruyter Studies in Mathematics 20, Berlin, 1995.

[3] M. Bożejko, M. Leinert and R. Speicher, Convolution and limit theorems for conditionally free random variables, Pacific J. Math. 175 (1996), 357-388.

[4] M. Bożejko and W. Bryc, On a class of free Lévy laws related to a regression problem, J. Funct. Anal. 236 (2006), 59-77. 
[5] T. Chihara, An Introduction to Orthogonal Polynomials, Mathematics and Its Applications 13, Gordon and Breach, New York 1978.

[6] M. Hinz and W. Młotkowski, Free cumulants of some probability measures, Banach Center Publications 78 (2007), 165-170

[7] A. D. Krystek and L. J. Wojakowski, Associative convolutions arising from conditionally free convolution, Infin. Dimens. Anal. Quantum Probab. Related Top. 8 (2005), 515-545.

[8] W. Młotkowski, Some class of polynomial hypergroups, Banach Center Publications 73 (2006), 357-362.

[9] W. Młotkowski and R. Szwarc, Nonnegative linearization for polynomials orthogonal with respect to discrete measures, Constructive Approximation 17 (2001), 413-429.

[10] N. Saitoh and H. Yoshida, The infinite divisibility and orthogonal polynomials with a constant recursion formula in free probability theory, Probability and Mathematical Statistics 21 (2001), 159-170.

[11] R. Szwarc, Orthogonal polynomials and a discrete boundary value problem, I, II, SIAM J. Math. Anal. 23 (1992), 959-969. 